\title{
Avanços no Tratamento Percutâneo das Doenças Cardíacas Estruturais
}

\author{
Áurea J. Chaves
}

0 tratamento percutâneo das doenças cardíacas estruturais (ou não-coronárias) se expande e se redefine a cada dia, e traz soluções para variados tipos de afecção. Entre essas afecções destacamse as comunicações intra e extracardíacas, incluindo os defeitos do septo atrial, o forame oval patente, os defeitos do septo ventricular, a persistência do canal arterial, as estenoses de artéria e veias pulmonares, a coarctação da aorta, a oclusão do apêndice atrial esquerdo (AAE), os defeitos valvares, a correção da regurgitação paraprotética, das fístulas arteriovenosas e das desordens protéticas vasculares, a ablação septal na cardiomiopatia hipertrófica idiopática obstrutiva, e a pericardiotomia por balão nas efusões crônicas. Em sintonia com esses avanços, esta edição da Revista Brasileira de Cardiologia Invasiva (RBCI) traz artigo que trata da oclusão do AAE em pacientes com fibrilação atrial de alto risco para acidente vascular cerebral e não candidatos à anticoagulação, e dois artigos que avaliam a evolução a médio prazo do implante transcateter de prótese valvular aórtica.

Armaganijan et al., do Instituto Dante Pazzanese de Cardiologia (São Paulo, SP), apresentam a experiência inicial da instituição com o dispositivo Amplatzer ${ }^{\mathrm{TM}}$ Cardiac Plug na oclusão do AAE, uma das poucas publicações a respeito na literatura mundial e a primeira no Brasil. Essa prótese e o dispositivo WATCHMAN são os dois oclusores aprovados para uso clínico na Europa. Nesse artigo os autores descrevem, com detalhes, os critérios de seleção dos pacientes, as características do novo dispositivo e as etapas do implante, guiadas por nova modalidade de imagem, a ecocardiografia tridimensional em tempo real. Essa técnica tem a capacidade de fornecer imagens simultâneas de cateteres e próteses, da afecção a ser tratada e das estruturas adjacentes. Os autores mostram também os resultados obtidos, explorando, inclusive, as dificuldades técnicas de um dos casos, no limite da indicação para o implante da prótese. Complementa o texto ampla documentação por imagem dos passos mais importantes do procedimento. Jilaihawi e Kar, do Cedars-Sinai Heart Institute (Los Angeles, Estados Unidos), em seu editorial, levantam importantes questões relativas ao fechamento do AAE, como a determinação da adequação clínica dos pacientes ao procedimento, com pontuação
CHADS2 apropriada, a importância da avaliação anatômica detalhada do $\mathrm{AAE}$, que apresenta grande variabilidade de tamanho inter e intrapaciente, sua preferência pelo ecocardiograma transesofágico em tempo real, especialmente com os recursos da modalidade tridimensional como método de eleição para monitorizar o procedimento, e a importância da curva de aprendizado dos operadores na redução de complicações periprocedimento.

Lemos et al., do Hospital Sírio-Libanês (São Paulo, $\mathrm{SP})$, trazem a experiência conjunta desse centro e da Universidade de Bolonha, na Itália, sobre o implante transcateter de CoreValve, no qual pacientes com perfil clínico característico - idosos, de alto risco cirúrgico - demonstram resultados hospitalares e em um ano bastante promissores. Bernardi et al., do Instituto de Cardiologia/Fundação Universitária de Cardiologia (Porto Alegre, RS), acrescentam em seu artigo importantes informações ecocardiográficas, obtidas aos dois anos de evolução, dos primeiros pacientes pertencentes à experiência inicial do Sul do Brasil tratados com CoreValve. Demonstram em seus pacientes a durabilidade dos resultados e os benefícios em termos de redução progressiva da massa ventricular esquerda, ao longo desse período de observação. Siqueira, Abizaid e Sousa, do Instituto Dante Pazzanese de Cardiologia (São Paulo, SP), em editorial correspondente, abordam os avanços relacionados ao implante transcateter de prótese valvular aórtica, como a redução do perfil da prótese, a utilização de vias de acesso alternativas, o aperfeiçoamento da técnica e a normatização dos desfechos pelo Valve Academic Research Consortium (VARC). Lembram também os desafios a serem vencidos, como a insuficiência aórtica paraprotética, o acidente vascular cerebral isquêmico e a necessidade de marca-passo definitivo.

Esta edição traz uma novidade: a publicação de artigo que descreve o desenho de um estudo, à semeIhança do que ocorre há anos no periódico American Heart Journal. Nesse artigo os autores descrevem o protocolo do estudo, incluindo as bases científicas que justificam a hipótese a ser investigada, a metodologia e a análise de dados que será empregada. Serão aceitos pela $\mathbf{R B C I}$, para julgamento entre os pares, somente estudos multicêntricos já aprovados pela Agência Na- 
cional de Vigilância Sanitária (Anvisa). O artigo que inaugura essa modalidade de publicação, dos investigadores do Registro eNOBORI - Brasil, descreve o racional e o desenho do estudo, com a finalidade de avaliar o desempenho do stent coronário com liberação de biolimus A9 Nobori $^{\mathrm{TM}}$ em casos de complexidades variadas, com a análise de subgrupos pré-especificados em diversos centros nacionais.

Para finalizar, comunicamos a completa reestruturação do conteúdo do portal da $\mathbf{R B C l}$, incluindo a missão do periódico e as novas "Instruções aos Autores", inspiradas em publicações internacionais de cardiologia, em especial na Revista Española de Cardiología e no JACC Cardiovascular Interventions. São instruções completas e objetivas, a maioria já observada pela $\mathbf{R B C I}$, em que são enfatizados: o ineditismo do manuscrito, com a obrigatoriedade do envio da De- claração de Transferência de Direitos Autorais do artigo aceito para publicação; os aspectos éticos da pesquisa, que deve ser aprovada pelo Comitê de Ética da instituição em que foi conduzida; e a Declaração de Conflito de Interesses do primeiro autor e dos demais autores, que deve constar tanto na carta de submissão do manuscrito à Revista como na primeira página do artigo. Complementam o documento instruções específicas para cada uma das categorias de artigos aceitos para publicação, em especial sobre a estrutura do manuscrito, com limites para número de palavras, autores (exceto para artigos originais), referências, tabelas e figuras. Esperamos, dessa maneira, aprimorar ainda mais a qualidade dos manuscritos publicados na RBCI.

Boa leitura a todos!

Áurea J. Chaves Editora 